\title{
Critical Evaluation!
}

Fachgesellschaften obliegen die Forschung und deren Umsetzung in die Praxis. Dazu gehören auch die Garantie der Qualität, und die Kontrolle von Nutzen und Gefahren. Fachzeitschriften unterstützen dies durch die Dokumentation der Forschung und durch die Verbreitung gewonnener Einsichten und Möglichkeiten für die Praxis und zum Wohle der Patienten. So auch unsere Zeitschrift. Dies betrifft im Besonderen die „Dauerbrenner“, Problemfelder, deren Nutzen, Risiken und Schäden immer wieder überprüft und zu Recht gerückt gehören. So ein Bereich ist die Abschätzung der biopositiven und bionegativen Effekte der UVStrahlung auf den Menschen, sowohl der Sonne als auch von künstlichen Lichtquellen (Akt Dermatol 2005; 31: 351-353).

Ein anderes, weites Feld betrifft Nutzen und Grenzen von Allergietestungen und deren Indikation. Solches geschieht im Positionspapier zur Formulierung von Leitlinien (JDDG 2006; 4: 72-85) oder durch gezielte Stellungnahme (SÄZ 2005; 86: 1565 - 1568). Und auch die Komplementärmedizin, deren Wirksamkeit in der Schweiz auf politische Verordnung hin evaluiert wurde. Es war ein strittiges Unterfangen (SÄZ 2006; 87: 25 -28), in welchem, wie wäre es anders zu erwarten gewesen, die Wirksamkeit der evaluierten Bereiche der Komplementärmedizin die Spielbreite der Placebo-Effekte nicht wesentlich überschritt.

Also die Komplementärmedizin rechtfertigt sich nicht über naturwissenschaftliche Beweisführung. Ihre Argumente liegen im affektiven Bereich, wie Umsatz, Nachfrage, Akzeptanz und über Verknüpfungen mit „ganzheitlichen Systemen“, mit spiritualistischen Ansätzen, mit uralten fernöstlichen Traditionen oder mit der Umgehung der modernen, rationalen, naturwissenschaftlichen Medizin und deren apparativen Komponente.
Man kann das verstehen. Die Hirnforschung ordnet dem älteren Hirnanteil, welcher das limbische System enthält, die Qualitäten Emotion, Motivation, das Vegetativum und das Irrationale zu, eingeschlossen Gedächtnis und Verarbeitung dieser Leistungen. Das jüngere Großhirn befähigt den Menschen zur Erbringung rationaler, naturwissenschaftlicher Leistungen, welche sich seit der Aufklärung in der Wissenschafts- und Kulturwelt massiv durchsetzen. Viele Menschen sind versucht, die emotionale Welt zu verdrängen. Dies zeigt sich z. B. am „Abwählen der Religion“. Aber das alte Gehirn ist nicht verkümmert, es fordert sein Recht, macht sich bemerkbar und äußert sich durch die Wünsche nach Spiritualität, „Ganzheit“, Behütung und es sucht sich „Heil“ in uralten Strukturen. Und vom „Heil zum Heiler“ wird auf ursprüngliche Krankheitsvorstellungen zurückgegriffen. Der rationalen Heilkunst wird misstraut. Zwangsläufig kommt man zum Thema Bioresonanz, wobei versucht wird, eine komplementärmedizinische Methode mit naturwissenschaftlichen, physikalischen Grundlagen zu argumentieren. Dabei wird die Physik falsch ausgelegt und die Messungen zur Diagnostik sowie die therapeutischen Anwendungen erbringen keineswegs das, was postuliert wird. Dies wird in der Stellungnahme der Fachkommission der Schweizerischen Gesellschaft für Allergologie und Immunologie (SGAI) klar dargelegt und als Unsinn bezeichnet (SÄZ 2006; 87: 50 - 54). Wir sind dankbar für die Genehmigung, die Stellungnahme in dieses Heft zu übernehmen (Seite 73-77) und unseren Lesern zukommen zu lassen. Zu Recht schreibt Herr Kollege Bruno Kesseli, Chefredaktor der SÄZ: „Wenn eine medizinische Methode - ob schul- oder komplementärmedizinischer Art - sich auf naturwissenschaftliche Grundlagen beruft, muss sie sich auch am aktuellen Stand des Wissens messen lassen“" (SÄZ 2006; 87, 128). Und da ist sie durchgefallen, die Bioresonanz! 